\title{
Epithelial-mesenchymal transition-like events in vulvar cancer and its relation with HPV
}

\author{
I S Rodrigues ${ }^{1}$, A M Lavorato-Rocha ${ }^{1}$, B de M Maia ${ }^{1}$, M M A Stiepcich ${ }^{2}$, F M de Carvalho ${ }^{1}$, \\ $\mathrm{G}_{\text {Baiocchi }}{ }^{3}$, F A Soares ${ }^{1}$ and R M Rocha ${ }^{1}$ \\ ${ }^{1}$ Department of Anatomic Pathology, AC Camargo Cancer Center, Rua Professor Antônio Prudente, 211, 1 andar, prédio Hilda \\ Jacob, Liberdade-São Paulo 01509 010, Brazil; ²Department of Anatomic Pathology, Fleury Institute, Avenida General Valdomiro \\ de Lima 508, Jabaquara-São Paulo 04344 903, Brazil and ${ }^{3}$ Department of Gynecology Oncology, AC Camargo Cancer Center, Rua \\ Professor Antônio Prudente, 211, térreo, prédio Carmen Prudente, Liberdade-São Paulo 01509 010, Brazil
}

Background: Epithelial-to-mesenchymal transition (EMT) still remains an obscure event in vulvar squamous cell carcinoma (VSCC).

Methods: Immunohistochemistry (IHC) expression of E-cadherin, $\beta$-catenin, Snail, Slug, Twist and Vimentin was analysed in 87 VSCC, controlled for human papillomavirus (HPV) positivity, considering tumour front and central tumour as different morphological categories from the same tumour.

Results: Lower $\beta$-catenin and higher Vimentin expression was associated with invasive front when compared with the central tumour $(P=0.013$ and $P \leqslant 0.001$, respectively). Higher expression of E-cadherin in central tumour was significantly related to absence of vascular and perineural invasion, lower invasion depth and $\leqslant 2$ lymph node involvement. Loss of $\beta$-catenin and high Slug, Snail and Twist expression was associated with HPV-negative tumours. Moreover, $\beta$-catenin lower expression associated with gain in Slug expression predicts a subgroup with worst outcome $(P=0.001)$. Lower expression of $\beta$-catenin in both central tumour and invasive front correlated with lower overall survival $(P=0.021$ and $P=0.011$, respectively). Also, multivariate analysis showed that lower $\beta$-catenin expression was independently associated with poorer outcome $(P=0.044)$.

Conclusion: Human papillomavirus-related tumours show better prognosis and outcome; besides, they do not progress through EMT phenomenon. Immunohistochemical analysis of $\beta$-catenin in invasive tumour front is a key issue for establishing prognosis of vulva cancer.

The incidence of vulvar cancer has risen steadily at $20 \%$ over the last 40 years (Lanneau et al, 2009) and represents nowadays the fourth most common type of gynaecological cancer, with an incidence of two to three per 100000 women yearly (van de Nieuwenhof et al, 2011). Vulvar squamous cell carcinoma (VSCC) comprises $70 \%$ of all cases of vulvar cancer and is primarily a disease of postmenopausal women with a mean age at diagnosis of $\sim 70$ years. Vulvar squamous cell carcinoma is usually diagnosed in a stage still amenable to potentially curative treatments, including surgery and/or radiation therapy with or without chemotherapy. However, several patients present metastatic disease at diagnosis, among those, 30-50\% will relapse (De Melo Maia et al, 2012; Santeufemia et al, 2012). Local or regional recurrences and distant metastasis have a critical role in progress of VSCC and the mechanism underlying their occurrence remains poorly understood.

During metastatic progression, important early events are loss of cell-cell adhesion detachment of tumour cells from the primary site (Cooke et al, 2012) and hypoxia that induces epithelial-tomesenchymal transition (EMT) of cells specifically by activation of master regulators of EMT, Twist and Slug (Sun et al, 2009), which are suggested to have an essential role in promoting metastasis (Cardiff, 2005).

E-cadherin-mediated cell adhesion requires intracellular attachment to the actin cytoskeleton by interaction with catenins, such as $\beta$-catenin, that may also act as an oncoprotein, becoming one of the key downstream effectors in the Wnt-Akt signalling pathway. In normal epithelial cells, $\beta$-catenin is localised at the cell

*Correspondence: Dr RM Rocha; E-mail: rafael.malagoli@gmail.com

Received 12 February 2013; revised 9 May 2013; accepted 14 May 2013; published online 18 June 2013

(c) 2013 Cancer Research UK. All rights reserved 0007-0920/13 
membrane. The unbound $\beta$-catenin is degraded by the ubiquitinproteasome system that involves the glycogen synthase kinase 3 beta. Stabilisation of cytoplasmic $\beta$-catenin by aberrant activation of Wnt signalling leads to its accumulation, complexed with lymphoid enhancer factor/Tcf (LEF/Tcf) transcription factors and transactivation of LEF/Tcf target genes. Activation of these genes leads to cell proliferation and inhibition of apoptosis (Bhangu et al, 2012). Some studies reported that reduced assembly of membranous $\beta$-catenin induces upregulation of Slug and the mesenchymal marker, Vimentin, key markers for EMT characterisation (Peinado et al, 2007; Heuberger and Birchmeier, 2010).

There are few studies assessing the impact of EMT markers on the progression of VSCC, and it remains largely unknown whether these markers have significance in the prognosis of this type of cancer. Therefore, considering VSCC morphological heterogeneity, evaluating the expression of EMT-related protein set in the tumour invasion may bring a novel perspective in establishing prognosis of patients with vulvar cancer, and also in the understanding of VSCC tumour biology. In this retrospective study, we evaluated the expression of E-cadherin, $\beta$-catenin, Snail, Slug, Twist and Vimentin in VSCC at the invasive front and central tumour, and associated their expression with clinical data collected from patient's medical records and the presence or not of human papillomavirus (HPV) infection.

\section{MATERIALS AND METHODS}

Sample selection. Eighty-seven cases of VSCC diagnosed between 1979 and 2006 were selected from the Anatomic Pathology Department of AC Camargo Cancer Center, Brazil. In situ carcinoma and patients subjected to preoperative chemotherapy and radiotherapy were excluded. Original H\&E slides were reviewed by experienced pathologist (MMAS) in order to confirm diagnosis and select the most suitable paraffin-embedded tissue for further studies in whole sections. Clinical and follow-up information were obtained up to 5 years after diagnosis for all patients.

\section{HPV DNA detection and genotyping}

DNA extraction. Total DNA was extracted from up to eight tissue sections (10- $\mu$ m-thick). Sections were pretreated with $350 \mu \mathrm{l} \mathrm{ATL}$ lysis buffer from the DNA FFPE kit (Qiagen, Valencia, CA, USA), added directly to the paraffin sections, followed by incubation of the tightly closed tubes at $120^{\circ} \mathrm{C}$ for $20 \mathrm{~min}$. All procedures followed the supplier's specification. DNA was then quantified at NanoDrop ND-1000 spectrophotometer (Wilmington, DE, USA) and analysed on $1 \%$ agarose gel.

HPV genotyping. Human papillomavirus detection and typing was performed using the linear array HPV-genotyping test (Roche Molecular Systems; Branchburg, NJ, USA). The assay is based on L1 consensus PCR with PGMY primers yielding a 450-bp amplicon, with type-specific hybridisation to detect 37 individual types $(6,11,16,18,26,31,33,35,39,40,42,45,51,52,53,54,55$,
$56,58,59,61,62,64,66,67,68,69,70,71,72$ 73, 81, 82, 83, 84, 89 and IS39). In addition, the assay includes $\beta$-globin primers (150-bp amplicon) detected on the HPV typing strip as positive control for amplifiable sample DNA. Linear array HPV-genotyping strips were manually interpreted using the HPV reference guide provided. The products of hybridisation were detected by a colour reaction with alkaline phosphatase-streptavidin conjugate and substrate (5-bromo-4-chloro-3-indolyl phosphate and nitrobluetetrazolium), which results in a purple precipitate. Hybridisation results were visually assessed by comparison with the standard grid.

Immunohistochemistry. Immunohistochemistry (IHC) was performed in whole sections (4- $\mu \mathrm{m}$-thick) of formalin-fixed paraffinembedded using a Ventana Benchmark XT automated stainer (Ventana Medical Systems; Tucson, AZ, USA). Table 1 presents the primary antibodies used in this study. All IHC-stained slides were digitised and analysed using a whole-slide imaging system (ScanScope XT; Aperio Technologies; San Diego, CA, USA; Table 3). All slides were scanned at $0.25 \mu \mathrm{m}$ per pixel resolution, and the images saved in the password-protected database (Spectrum version 10.2.2.2314) provided by Aperio via web-accessible server.

Scoring of IHC expression. All sections were blindly analysed and the expression pattern was evaluated in a quantitative manner, whereby the levels of expression are represented by the percentage of positive cells and the intensity of immunostaining (HSCORE = $\Sigma(\mathrm{i} \times \mathrm{Pi})$, where $\mathrm{Pi}=$ percentage of positive pixels, varied from 0 to $100 \%$ and pixel staining intensity $i=0,1,2$ or 3 ), with a ranking between 0 and 300 (Alvarenga et al, 2013).

Two areas of each tumour - central tumour and invasion front were morphologically selected and assessed for expression of each protein. Concerning central tumour, it was considered as the largest extension tumour area, and at least three fields of each section were randomly selected and analysed. Invasion front, defined as single cells or clusters of up to five cells detached from the main tumour mass that broadly infiltrate the adjacent stroma (Paterson et al, 2013), was evaluated in 10 different randomly selected fields.

The levels of immunoreactivity for all markers were established as numerical variables. Membrane $\beta$-catenin expression was assigned as negative (0) or positive (1) by the software.

Clinicopathological parameters of the patients. The clinical data obtained from the medical records were: age and 5-year follow-up. Pathological data, such as lymph node involvement, pathological stage (FIGO staging), inflammatory infiltration, presence of vascular and perineural invasion, associated lesions (differentiated and high-grade VIN, lichen sclerosus and squamous hyperplasia) and invasion depth were evaluated.

Statistics analysis. The Statistical Package for the Social Sciences (SPSS, Upper Saddle River, NJ, USA) 17.0 software was used. The association between $\beta$-catenin and clinicopathological variables was analysed with the $\chi^{2}$-test for categorical data and Fisher's exact test was used for small numbers. Comparison of the protein expression between the central tumour and invasion front was performed using Wilcoxon signed-rank test. Mann-Whitney test

Table 1. Protein, clones, supplier and dilution of the primary antibodies used in the immunohistochemical assays

\begin{tabular}{|l|l|l|c|}
\hline Antibody & Clone & Supplier & Dilution \\
\hline E-cadherin & $36 /$ E-cadherin & BD Biosciences, Franklin Lakes, NJ, USA & $1 / 2000$ \\
\hline$\beta$-catenin & 17 C2 & NeoMarkers Biotechnology, Fremont, CA, USA & $1 / 50$ \\
\hline Snai1 & A242 & Bioworld Technology, Minneapolis, MN, USA & $1 / 200$ \\
\hline Slug & C1967 & Cell Signaling, Beverly, MA, USA & $1 / 100$ \\
\hline Twist2 & 3 C8 & Abnova, Jhongli, Taiwan & $1 / 25$ \\
\hline Vimentin & V9 & Dako, Carpinteria, CA, USA & $1 / 1500$ \\
\hline
\end{tabular}


Table 2. Clinicopathological data and HPV infection

\begin{tabular}{|c|c|c|c|}
\hline Characteristic Clinicopathological & ValueN (\%) & N/total HPV positive (\%) & $P$-value \\
\hline $\begin{array}{l}\text { Age (years), median (range) } \\
46-49 \text { years old } \\
\geqslant 50 \text { years old }\end{array}$ & $\begin{array}{l}68.75(46-90) \\
30(34.5) \\
57(65.5)\end{array}$ & $\begin{array}{l}- \\
11 / 34(32.3) \\
23 / 34(67.7)\end{array}$ & $0 . \overline{0}$ \\
\hline \multicolumn{4}{|l|}{ Histologic types } \\
\hline $\begin{array}{l}\text { Grade } 1 \text { SCC } \\
\text { Grade } 2 \text { SCC } \\
\text { Grade } 3 \mathrm{SCC} \\
\text { Basaloid } \mathrm{Ca} \\
\text { Verrucous } \mathrm{Ca}\end{array}$ & $\begin{array}{c}27(31) \\
46(52.9) \\
8(9.2) \\
4(4.6) \\
2(2.3)\end{array}$ & $\begin{array}{c}9 / 34(26.5) \\
13 / 34(38.2) \\
5 / 34(14.7) \\
4 / 34(11.8) \\
0\end{array}$ & $\begin{array}{l}- \\
- \\
- \\
-\end{array}$ \\
\hline \multicolumn{4}{|l|}{ Inflammatory infiltrate } \\
\hline $\begin{array}{l}\text { No } \\
\text { Yes }\end{array}$ & $\begin{array}{l}79(90.8) \\
8(9.2)\end{array}$ & $\begin{array}{l}16 / 57(28.1) \\
29 / 40(72.5)\end{array}$ & 0.951 \\
\hline \multicolumn{4}{|l|}{ Vascular/perineural invasion } \\
\hline $\begin{array}{l}\text { No } \\
\text { Yes }\end{array}$ & $\begin{array}{l}73(84) \\
14(13)\end{array}$ & $\begin{array}{l}47 / 56(83.9) \\
4 / 38(10.5)\end{array}$ & 0.445 \\
\hline \multicolumn{4}{|l|}{ Lymph node involvement } \\
\hline $\begin{array}{l}\leqslant 2 \\
>2 \\
\text { No }\end{array}$ & $\begin{array}{l}80(92) \\
2(2.3) \\
5(5.7)\end{array}$ & $\begin{array}{c}28 / 30(93.3) \\
2 / 23(8.7)\end{array}$ & 1.000 \\
\hline \multicolumn{4}{|l|}{ Associated lesions } \\
\hline $\begin{array}{l}\text { VIN } \\
\text { Lichen sclerosus and squamous hyperplasia } \\
\text { No }\end{array}$ & $\begin{array}{l}25(29) \\
8(9) \\
54(62)\end{array}$ & $\begin{array}{c}8 / 22(36.4) \\
4 / 10(40)\end{array}$ & 0.683 \\
\hline \multicolumn{4}{|l|}{ Invasion depth } \\
\hline $\begin{array}{l}\text { Superficial and mid dermis } \\
\text { Deep dermis and subcutis }\end{array}$ & $\begin{array}{l}37(42.5) \\
50(57.5)\end{array}$ & $\begin{array}{l}32 / 59(54.2) \\
16 / 40(40)\end{array}$ & 0.570 \\
\hline \multicolumn{4}{|l|}{ FIGO stage } \\
\hline $\begin{array}{l}\text { I and II } \\
\text { III and IV }\end{array}$ & $\begin{array}{l}56(64.4) \\
31(35.6)\end{array}$ & $\begin{array}{l}30 / 55(54.5) \\
12 / 38(31.6)\end{array}$ & 0.179 \\
\hline
\end{tabular}

was employed for interpretation of the association between protein expression and clinical parameters, and $P \leqslant 0.05$ was considered statistically significant for all tests. Kaplan-Meier curves were generated for protein expression in overall survival. The log-rank test was applied to test the significance of differences between stratified survival functions. Cox proportional-hazards regression analysis was performed to test the statistical independence and significance between pathological, molecular and clinical variables.

\section{RESULTS}

Sample characteristics and HPV Infection. Clinicopathological data of all patients are provided in Table 2. Local recurrence was observed in 25 out of 87 (28.7\%) patients. Human papillomavirus infection was detected in 34 out of 87 (39.1\%) cases. The most frequently detected high-risk HPV types were HPV16 (35.3\%), followed by HPV33 (8.9\%), HPV18 and HPV35 (both 5.9\%). Human papillomavirus coinfection was found in eight cases (23.5\%; Table 3). There was no association between HPV infection and vascular and perineural invasion, lymph node involvement, FIGO stage, invasion depth and presence of associated lesion (Table 2). However, HPV-positive cases showed better overall survival than the HPV-negative ones (Figure 1).

\section{Table 3. HPV type in patients with vulvar carcinoma}

\begin{tabular}{|c|c|}
\hline HPV type & $\mathbf{N}(\%)$ \\
\hline Positive cases & $34(39.1)$ \\
\hline \multicolumn{2}{|l|}{ Type HPV } \\
\hline $\begin{array}{l}\text { HPV } 16 \\
\text { HPV } 18 \\
\text { HPV } 33 \\
\text { HPV } 35 \\
\text { HPV } 45 \\
\text { HPV } 53 \\
\text { HPV } 71\end{array}$ & $\begin{aligned} & 12(35.3) \\
& 2(5.9) \\
3 & (8.9) \\
2 & (5.9) \\
1 & (2.9) \\
4 & (11.8) \\
2 & (5.9)\end{aligned}$ \\
\hline \multicolumn{2}{|l|}{ Coinfection } \\
\hline $\begin{array}{l}\text { HPV } 16+\text { HPV } 18+\text { HPV } 33 \\
\text { HPV } 16+\text { HPV } 33 \\
\text { HPV } 16+\text { HPV } 33+\text { HPV } 35 \\
\text { HPV } 16+\text { HPV } 33+\text { HPV } 84 \\
\text { HPV } 18+\text { HPV } 33+\text { HPV } 35 \\
\text { HPV } 31+\text { HPV } 33+\text { HPV } 82 \\
\text { HPV } 42+\text { HPV } 54\end{array}$ & $\begin{array}{l}1(2.9) \\
2(5.9) \\
1(2.9) \\
1(2.9) \\
1(2.9) \\
1(2.9) \\
1(2.9)\end{array}$ \\
\hline
\end{tabular}


E-cadherin expression. Immunoexpression for E-cadherin was considered as membrane staining; it was positive at the central tumour and invasive front of 47 (54\%) and 45 (51.7\%) cases, respectively, (Figure 2A and D). There was no difference of E-cadherin expression between central tumour and invasive front $(P=0.692$; Figure $2 \mathrm{G})$. Lower expression of E-cadherin at the central tumour was significantly related to vascular and perineural invasion, invasion depth and $\geqslant 2$ lymph node involvement (Table 4A). Similarly, lower expression of E-cadherin at the tumour invasive front was related with vascular and perineural invasion, and FIGO stage III/IV (Table 4B). On the other hand, there was no relation between E-cadherin expression and HPV

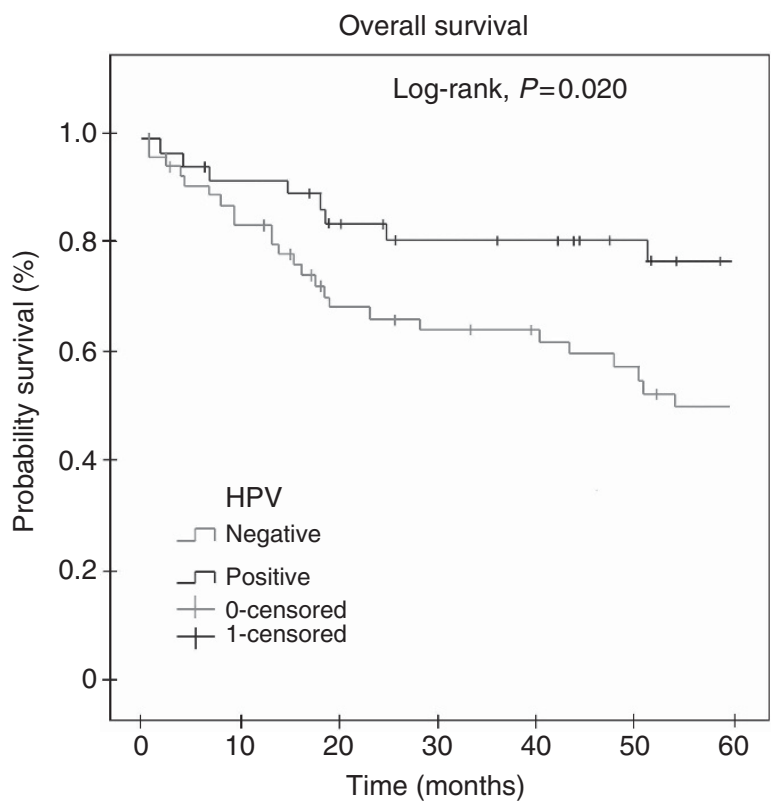

Figure 1. Overall survival curve of patients according to HPV infection. Shorter overall survival was observed for HPV-negative cases $(P=0.020)$. Kaplan-Meier method, log-rank test. infection (Table 5), inflammatory infiltrate, associated diseases, and overall and disease-free survival (Supplementary Table S1).

$\beta$-catenin expression. Immunoexpression for $\beta$-catenin was specifically well localised in cell membrane, and positive in central tumour and invasion front in 50 out of 87 (57.5\%) and 40 out of 87 $(46 \%)$, respectively, (Figures $2 \mathrm{~B}$ and E). $\beta$-catenin was significantly less expressed at the invasive front when compared with central tumour $(P=0.013)$ (Figure $2 \mathrm{H})$. Nuclear $\beta$-catenin expression was not detected at all. There was no relation between $\beta$-catenin positivity and vascular and perineural invasion, invasion depth, lymph node involvement, associated lesions and inflammatory infiltration (Table 6). In contrast, loss of $\beta$-catenin expression at the central tumour and invasive front was significantly associated with FIGO stages III/IV cases $(P=0.016$ and $P=0.023$; Table 6) and absence of HPV infection $(P=0.001$ and $P=0.000$; Table 7).

Patients with lower expression of $\beta$-catenin in both central tumour and invasive front presented lower overall survival $(P=0.021$ and $P=0.011$, respectively; Figure $3 \mathrm{~A}$ and $\mathrm{B})$. Concerning multivariate analysis, lower expression of $\beta$-catenin in invasive front was independently associated with lower overall survival $(P=0.044$; Table 8$)$.

Vimentin expression. Vimentin expression presented as specific, sharp and well-localised cytoplasmic immunostaining pattern, being positive in 45 out of 87 cases $(51.7 \%)$ at the central tumour and 44 out of 87 cases $(50.6 \%)$ at the invasive front (Figure $2 \mathrm{C}$ and $\mathrm{F}$ ). There was a significant difference in Vimentin expression between invasive front and central tumour $(P<0.001$; Figure 2I). Vimentin positivity was also significantly associated with invasion depth $(P=0.017$; Table 4A). Conversely, there was no association between Vimentin and vascular and perineural invasion, lymph node involvement, inflammatory infiltration, associated lesions, overall, disease-free survival (Supplementary Table S1 and Table 4B) and HPV infection (Table 5).

Slug expression. Slug expression presented as specific, sharp and well-localised nuclear staining pattern, with variable staining intensity degree along the same tumour. Slug expression was observed at the central tumour and invasive front in 42 out of 87 cases (48.2\%) and 44 out of 87 cases (50.6\%), respectively,

Table 4A. E-cadherin, Snail, Twist and Vimentin expression and clinicopathological data in central tumour

\begin{tabular}{|c|c|c|c|c|c|c|}
\hline Protein expression & Variables & Category & Quartile1 & Median & Quartile 3 & $\boldsymbol{P}$-value \\
\hline \multirow[t]{6}{*}{ E-cadherin } & Vascular/perineural invasion & No & 110.70 & 150.713 & 172.44 & $0.010^{a}$ \\
\hline & & Yes & 37.40 & 89.47 & 144.50 & \\
\hline & Invasion depth & SMD & 117.97 & 157.17 & 175.96 & $0.001^{\mathrm{a}}$ \\
\hline & & DDS & 60.90 & 117.00 & 161.66 & \\
\hline & Lymph node involvement & $\leqslant 2$ & 94.47 & 139.67 & 169.37 & $0.044^{a}$ \\
\hline & & $>2$ & 28.308 & 56.06 & 72.620 & \\
\hline \multirow[t]{4}{*}{ Snail } & Vascular/perineural invasion & No & 1.58 & 6.31 & 28.0 & $0.031^{a}$ \\
\hline & & Yes & 0.64 & 1.74 & 7.37 & \\
\hline & Invasion depth & SMD & 1.73 & 8.26 & 37.00 & $0.029^{a}$ \\
\hline & & DDS & 0.73 & 2.17 & 7.54 & \\
\hline \multirow[t]{2}{*}{ Twist 2} & Associated lesions & VIN & 30.32 & 76.55 & 117.26 & $0.014^{a}$ \\
\hline & & $\mathrm{LH}$ & 0 & 17.60 & 46.72 & \\
\hline \multirow[t]{2}{*}{ Vimentin } & Invasion depth & SMD & 21.88 & 35.59 & 52.42 & $0.017^{a}$ \\
\hline & & DDS & 13.97 & 24.19 & 38.85 & \\
\hline
\end{tabular}


Table 4B. E-cadherin, Slug, Snail, Twist2 and Vimentin expression and clinicopathological factors at the invasive front

\begin{tabular}{|c|c|c|c|c|c|c|}
\hline Protein expression & Variables & Category & Quartile 1 & Median & Quartile 3 & $\boldsymbol{P}$-value \\
\hline \multirow[t]{12}{*}{ E-cadherin } & Vascular/perineural invasion & No & 109.92 & 147.06 & 173.2 & $0.044^{a}$ \\
\hline & & Yes & 49.53 & 138.96 & 148.41 & \\
\hline & FIGO stage & $|-| \mid$ & 121.44 & 149.91 & 174.86 & $0.009^{a}$ \\
\hline & & III-IV & 73.108 & 130.86 & 146.80 & \\
\hline & Invasion depth & SMD & 101.30 & 145.17 & 166.72 & 0.481 \\
\hline & & DDS & 116.27 & 141.73 & 161.05 & \\
\hline & Lymph node involvement & $\leqslant 2$ & 99.65 & 133.12 & 162.22 & 0.747 \\
\hline & & $>2$ & 76.399 & 141.76 & 148.54 & \\
\hline & Associated lesions & $\mathrm{VIN}$ & 81.49 & 144.41 & 165.09 & 0.887 \\
\hline & & LH & 108.33 & 135.98 & 168.33 & \\
\hline & Inflammatory infiltrate & No & 126.87 & 146.00 & 170.05 & 0.236 \\
\hline & & Yes & 91.97 & 140.78 & 157.72 & \\
\hline \multirow[t]{12}{*}{ Slug } & Vascular/perineural invasion & No & 1,204 & 4.69 & 18.24 & 0.991 \\
\hline & & Yes & 1.185 & 2.95 & 20.97 & \\
\hline & Invasion depth & SMD & 2.049 & 5.42 & 23.88 & 0.176 \\
\hline & & DDS & 0.619 & 2.76 & 13.23 & \\
\hline & Lymph node involvement & $\leqslant 2$ & 0.766 & 5.18 & 15.54 & 0.747 \\
\hline & & $>2$ & 1.352 & 1.50 & 12.48 & \\
\hline & Associated lesions & $\mathrm{VIN}$ & 2.43 & 4.37 & 23.66 & $0.029^{a}$ \\
\hline & & LH & 0.61 & 0.97 & 2.33 & \\
\hline & FIGO stage & $|-| \mid$ & 1.134 & 2.91 & 13.54 & 0.100 \\
\hline & & III-IV & 1.695 & 4.32 & 34.36 & \\
\hline & Inflammatory infiltrate & No & 1.06 & 2.79 & 14.69 & 0.236 \\
\hline & & Yes & 1.32 & 4.83 & 21.15 & \\
\hline \multirow[t]{12}{*}{ Snail } & Vascular/perineural invasion & No & 0.98 & 6.41 & 25.80 & 0.148 \\
\hline & & Yes & 0.19 & 2.28 & 10.97 & \\
\hline & Invasion depth & SMD & 0.57 & 6.41 & 29.72 & 0.511 \\
\hline & & DDS & 0.60 & 4.02 & 12.58 & \\
\hline & Lymph Node Involvement & $\leqslant 2$ & 1.01 & 5.89 & 33.89 & 0.081 \\
\hline & & $>2$ & 0 & 0 & 1.14 & \\
\hline & Associated lesions & VIN & 4.04 & 7.37 & 21.44 & 0.625 \\
\hline & & LH & 0.54 & 5.31 & 19.09 & \\
\hline & FIGO stage & $|-| \mid$ & 0.59 & 4.83 & 15.62 & 0.171 \\
\hline & & III-IV & 0.63 & 5.33 & 33.92 & \\
\hline & Inflammatory infiltrate & No & 0.39 & 5.07 & 23.92 & 0.510 \\
\hline & & Yes & 0.68 & 5.26 & 22.62 & \\
\hline \multirow[t]{12}{*}{ Twist } & Vascular/perineural invasion & No & 0.28 & 5.77 & 19.88 & 0.258 \\
\hline & & Yes & 0.34 & 3.11 & 9.10 & \\
\hline & Invasion depth & SMD & 0.57 & 6.43 & 19.38 & 0.487 \\
\hline & & DDS & 0.10 & 3.58 & 9.35 & \\
\hline & Lymph Node Involvement & $\leqslant 2$ & 0.07 & 6.21 & 20.32 & 0.428 \\
\hline & & $>2$ & 1.65 & 3.31 & 5.01 & \\
\hline & Associated lesions & $\mathrm{VIN}$ & 53.74 & 77.11 & 145.22 & 0.052 \\
\hline & & LH & 0 & 18.87 & 74.97 & \\
\hline & FIGO stage & $|-| \mid$ & 0.05 & 5.10 & 9.68 & 0.856 \\
\hline & & III-IV & 0.58 & 5.00 & 20.32 & \\
\hline & Inflammatory infiltrate & No & 0.05 & 2.93 & 9.25 & 0.219 \\
\hline & & Yes & 0.67 & 6.17 & 18.54 & \\
\hline \multirow[t]{2}{*}{ Vimentin } & Vascular/perineural invasion & No & 40.52 & 68.14 & 105.91 & 0.287 \\
\hline & & Yes & 45.07 & 54.96 & 72.48 & \\
\hline
\end{tabular}


Table 4B. (Continued)

\begin{tabular}{|c|c|c|c|c|c|c|}
\hline Protein expression & Variables & Category & Quartile 1 & Median & Quartile 3 & $P$-value \\
\hline & Invasion depth & SMD & 45.83 & 68.86 & 102.43 & 0.148 \\
\hline & & DDS & 40.30 & 54.96 & 74.76 & \\
\hline & Lymph node involvement & $\leqslant 2$ & 40.54 & 58.94 & 79.89 & 0.617 \\
\hline & & $>2$ & 42.32 & 54.96 & 67.37 & \\
\hline & Associated lesions & VIN & 32.63 & 58.64 & 82.48 & 0.569 \\
\hline & & $\mathrm{LH}$ & 51.74 & 67.20 & 74.09 & \\
\hline & FIGO stage & $|-I|$ & 44.02 & 62.32 & 75.53 & 0.725 \\
\hline & & III-IV & 58.36 & 57.07 & 106.00 & \\
\hline & Inflammatory infiltrate & No & 43.48 & 66.91 & 75.98 & 0.676 \\
\hline & & Yes & 42.36 & 57.99 & 102.81 & \\
\hline $\begin{array}{l}\text { Abbreviations: } \leqslant 2=\text { two o } \\
\text { Gynecology and Obstetric } \\
\text { grade). Mann-Whitney tes } \\
{ }^{\text {a }} \text { Statistically significant, } P \leqslant\end{array}$ & $\begin{array}{l}\text { lymph node involvement; }>2= \\
=\text { lichen sclerosus and squamol } \\
\text { 87). }\end{array}$ & $\begin{array}{l}\text { ph node invol } \\
=\text { nuclear; SML }\end{array}$ & $\begin{array}{l}\text { = cytoplasmi } \\
\text { icial and minc }\end{array}$ & $\begin{array}{l}\text { eep dermis } \\
\mathrm{IN}=\text { vulvar }\end{array}$ & $\begin{array}{l}\text { utis; } F I G O=\text { Int } \\
\text { helial neoplasia }\end{array}$ & $\begin{array}{l}\text { nal Federatic } \\
\text { ntiated and }\end{array}$ \\
\hline
\end{tabular}

Table 5. E-cadherin, Slug, Snail, Twist and Vimentin expression according to the HPV infection

\begin{tabular}{|c|c|c|c|c|c|c|}
\hline $\begin{array}{l}\text { Protein expression } \\
\text { (immunopositivity) }\end{array}$ & Region & HPV infection & Quartile 1 & Median & Quartile 3 & $P$-value \\
\hline \multirow[t]{4}{*}{ E-cadherin } & Central tumour & No & 97.95 & 115.18 & 168.92 & 0.721 \\
\hline & & Yes & 73.451 & 131.49 & 175.50 & \\
\hline & Invasive front & No & 109.924 & 141.74 & 162.36 & 0.862 \\
\hline & & Yes & 88.476 & 145.22 & 158.95 & \\
\hline \multirow[t]{4}{*}{ Slug } & Central tumour & No & 2.127 & 8.09 & 31.95 & $0.018^{a}$ \\
\hline & & Yes & 0.690 & 2.11 & 8.66 & \\
\hline & Invasive front & No & 1.952 & 9.80 & 37.98 & $0.004^{a}$ \\
\hline & & Yes & 0.905 & 2.33 & 9.64 & \\
\hline \multirow[t]{4}{*}{ Snail } & Central tumour & No & 1.74 & 5.96 & 28.52 & 0.068 \\
\hline & & Yes & 0 & 1.96 & 15.50 & \\
\hline & Invasive front & No & 0.98 & 8.10 & 29.41 & $0.018^{a}$ \\
\hline & & Yes & 0.18 & 2.54 & 8.70 & \\
\hline \multirow[t]{4}{*}{ Twist } & Central tumour & No & 36.08 & 67.90 & 110.80 & $0.007^{a}$ \\
\hline & & Yes & 1.13 & 35.29 & 69.47 & \\
\hline & Invasive front & No & 0.88 & 6.67 & 20.33 & $0.009^{a}$ \\
\hline & & Yes & 0 & 2.10 & 8.43 & \\
\hline \multirow[t]{4}{*}{ Vimentin } & Central tumour & No & 16.03 & 30.04 & 48.02 & 0.486 \\
\hline & & Yes & 22.25 & 33.23 & 44.67 & \\
\hline & Invasive front & No & 44.54 & 64.24 & 103.00 & 0.389 \\
\hline & & Yes & 42.00 & 59.74 & 76.00 & \\
\hline
\end{tabular}

(Figure 4A and D). There was no difference of Slug expression at the invasive front when compared with the central tumour $(P=0.059$; Figure $4 \mathrm{G})$. Also, Slug expression was not associated with vascular and perineural invasion, lymph node involvement, FIGO stage, invasion depth, inflammatory infiltration, overall and disease-free survival (Supplementary Table S1 and Table 4B). Slug positivity was significantly associated with associated lesions, specially differentiated and high-grade VIN $(P=0.029$; Table 4B). Higher expression of Slug at the central tumour and invasive front was also most frequently observed in negative HPV cases $(P=0.018$ and $P=0.004$, respectively; Table 5).
Snail and Twist2 expression. Snail and Twist2 expression presented as sharp, well-localised nuclear and cytoplasmic staining. Snail was positive in both central tumour and invasive front in 44 out of 87 cases (50.6\%; Figure $4 \mathrm{~B}$ and E). Twist2 was positive at the central tumour and invasive front in 42 out of 87 (48.3\%) and 44 out of 87 cases (50.6\%; Figure 4C and Figure $4 \mathrm{~F}$ ). There was no difference in Snail expression at invasive front when compared with central tumour $(P=0.813$; Figure $4 \mathrm{H})$. Snail expression in central tumour was related to vascular and perineural invasion, invasion depth and associated lesions $(P=0.031, P=0.029$ and $P=0.018$; Table 4A); on the other hand, Snail expression at the 


\begin{tabular}{|c|c|c|c|c|c|c|}
\hline & & & Protein expression & & Protein expression & \\
\hline Variables & Category & Region & $\begin{array}{l}\beta \text {-catenin } \\
N / \text { total (\%) }\end{array}$ & $\boldsymbol{P}$-value & $\begin{array}{c}\beta \text {-catenin }{ }^{\text {low }} / \text { Slug }^{\text {high }} \\
N / \text { total }(\%)\end{array}$ & $\boldsymbol{P}$-value \\
\hline \multirow{4}{*}{$\begin{array}{l}\text { Vascular/perineural } \\
\text { invasion }\end{array}$} & No & IF & $34 / 65(52.3)$ & 0.293 & $16 / 65(24.6)$ & 0.293 \\
\hline & Yes & & 13/19 (68.4) & & $7 / 19$ (36.8) & \\
\hline & No & $\mathrm{CT}$ & $28 / 65(43.1)$ & 0.740 & $17 / 65(26.2)$ & 0.364 \\
\hline & Yes & & 9/19 (47.4) & & 7/19(36.8) & \\
\hline \multirow[t]{4}{*}{ Invasion depth } & SMD & IF & $25 / 50(50.0)$ & 0.701 & $14 / 50(28.0)$ & 0.701 \\
\hline & DDS & & $22 / 37(59.5)$ & & 9/37 (24.3) & \\
\hline & SMD & CT & $20 / 50(40.0)$ & 0.579 & $12 / 50(24.0)$ & 0.384 \\
\hline & DDS & & $17 / 37(45.9)$ & & 12/37 (32.4) & \\
\hline \multirow{4}{*}{$\begin{array}{l}\text { Lymph node } \\
\text { involvement }\end{array}$} & $\leqslant 2$ & IF & $23 / 42(54.8)$ & 0.870 & $12 / 42(28.6)$ & 1.000 \\
\hline & $>2$ & & $2 / 3(66.7)$ & & $1 / 3(33.3)$ & \\
\hline & $\leqslant 2$ & CT & $21 / 42(50.0)$ & 1.000 & $12 / 42(28.6)$ & 1.000 \\
\hline & $>2$ & & $2 / 3(66.7)$ & & $1 / 3(33.3)$ & \\
\hline \multirow[t]{4}{*}{ Associated lesions } & $\mathrm{VIN}$ & IF & $4 / 10(40.0)$ & 0.446 & $8 / 27(78.5)$ & 0.637 \\
\hline & LH & & $12 / 23(52.2)$ & & $2 / 5(38.9)$ & \\
\hline & $\mathrm{VIN}$ & CT & $3 / 10(30)$ & 0.438 & $6 / 22(41.7)$ & 0.473 \\
\hline & LH & & $8 / 23(34.8)$ & & $4 / 10 / 20(75)$ & \\
\hline \multirow[t]{4}{*}{ FIGO stage } & $|-| I$ & $\mathrm{IF}$ & $23 / 52(44.2)$ & $0.023^{a}$ & 9/52 (17.3) & $0.023^{a}$ \\
\hline & III-IV & & $21 / 30(70.0)$ & & $12 / 30(40.0)$ & \\
\hline & $|-| \mid$ & CT & 17/52 (32.7) & $0.016^{a}$ & 10/52 (19.2) & $0.041^{a}$ \\
\hline & III-IV & & $18 / 30(60.0)$ & & $12 / 30(40.0)$ & \\
\hline \multirow[t]{4}{*}{ Inflammatory infiltrate } & No & IF & $16 / 28(57.1)$ & 0.466 & $6 / 28(21.4)$ & 0.466 \\
\hline & Yes & & $31 / 59(52.5)$ & & 17/59 (28.8) & \\
\hline & No & CT & $11 / 28(39.3)$ & 0.673 & $6 / 28(21.4)$ & 0.376 \\
\hline & Yes & & $26 / 59(44.1)$ & & 18/59 (30.5) & \\
\hline \multicolumn{7}{|c|}{$\begin{array}{l}\text { Abbreviations: } \leqslant 2=\text { two or less lymph node involvement; }>2=\text { more than two lymph node involvement; } C T=\text { central tumour; } D D S=\text { deep dermis and subcutis; } F I G O=I n t e r n a t i o n a \\
\text { Federation of Gynecology and Obstetrics; IF=invasive front; } L H=\text { lichen sclerosus and squamous hyperplasia; } S M D=\text { superficial and mind dermis; VIN =vulvar intraepithelial neoplasi } \\
\text { (differentiated and high grade). } \chi^{2} \text { - and Fisher's exact test }(N=87) \text {. } \\
\text { a }\end{array}$} \\
\hline
\end{tabular}

invasive front was related to negative HPV cases $(P=0.018$; Table 5). There was also no difference in Twist 2 expression at the invasive front when compared with the central tumour $(P=0.113$; Figure 4I). However, Twist2 expression at the invasive front was associated with negative HPV cases $(P=0.009$ and $P=0.005$; Table 5). Besides, Twist 2 expression was also related to the presence of vulvar intraepithelial neoplasia differentiated $(P=0.014$; Table 4B). However, no association between Snail or Twist2 with lymph node involvement, inflammatory infiltration, FIGO stage, overall and disease-free survival was observed (Supplementary Table S1 and Table 4B).

Loss of expression of $\beta$-catenin together with high expression of Slug. Cases that expressed $\beta$-catenin ${ }^{\text {low }} /$ Slug $^{\text {high }}$ at the invasive front were related with FIGO stages III and IV $(P=0.023)$ and negativity for HPV $(P=0.000$; Table 7$)$. This pattern of expression $\left(\beta\right.$-catenin ${ }^{\text {low }} /$ Slug $\left.^{\text {high }}\right)$ was observed at the central tumour and invasive front in 24 out of $87(27.6 \%)$ and 21 out of 87 cases (24.1\%; Figure 5), and these patients had the poorest overall survival $(P=0.001$; Figure $3 \mathrm{C}$ and $\mathrm{D})$.

\section{DISCUSSION}

Alterations in cell adhesion are among the hallmark characteristics of a malignant epithelial tumour, including irregularities in expression and distribution of adhesion molecules, which is accompanied by degradation of extracellular matrix, and gain of mesenchymal cytoskeletal proteins such as N-cadherin, Vimentin, fibronectin and smooth muscle alfa-actin (Peinado et al, 2007; Thiery et al, 2009). Therefore, we investigated the expression of E-cadherin, $\beta$-catenin, Vimentin and the EMT markers Snail, Slug and Twist 2 in a large series of 87 cases of vulvar carcinoma. Also, concerning different morphological subpopulations of tumour cells, we evaluated the immunoexpression of these proteins in both central tumour and invasive front, in order to determine whether the expression of these molecules induce EMT-like, the acquisition of migratory and invasive behaviour and whether these events are related with HPV infection.

Loss of E-cadherin expression leads to reduced cell adhesiveness and detachment of tumour cells from each other, resulting in increased invasive potential of the neoplastic cells. The results of the present study reveal that there was statistical association between lower E-cadherin expression and vascular and perineural invasion, FIGO stages III and IV, deeper invasion and $\geqslant 2$ lymph node involvement (Table 4A and B). E-cadherin expression was not significantly reduced at the invasive front compared with the central tumour. Also, no association was found between decrease of E-cadherin expression, inflammatory infiltration and HPV infection (Table 5). Carico et al (2012) demonstrated that higher expression of E-cadherin was associated with specific subtypes of laryngeal cancer, such as in situ carcinoma, in which a strong and 


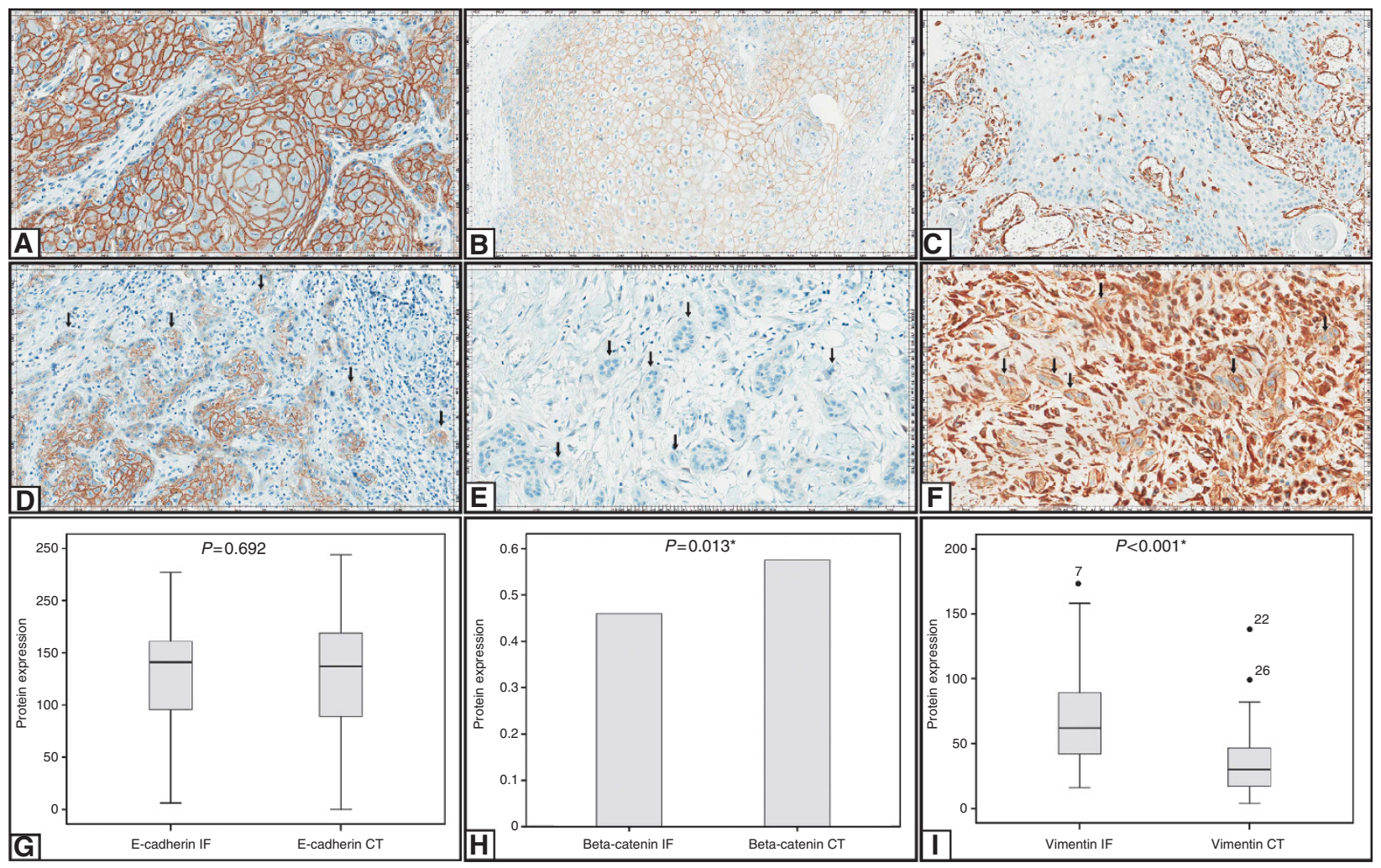

Figure 2. Immunohistochemical staining for E-cadherin, $\beta$-catenin and Vimentin is shown at central tumour and invasive front. Positive immunostaining for E-cadherin at central tumour (A) and invasive front (D); positive immunostaining for $\beta$-catenin at central tumour (B) and negative immunostaining for $\beta$-catenin at invasive front $(\mathbf{E})$; negative immunostaining for Vimentin at central tumour $(\mathbf{C})$ and positive immunostaining for vimentin at invasive front $(\mathbf{F})$. Comparison between the expression of E-cadherin $(\mathbf{G}), \beta$-catenin $(\mathbf{H})$ and Vimentin $(\mathbf{I})$ in central tumour and invasive front. All images were captured at $\times 200$ magnification. ${ }^{*}$ Statistical significance, $P \leqslant 0.05$.
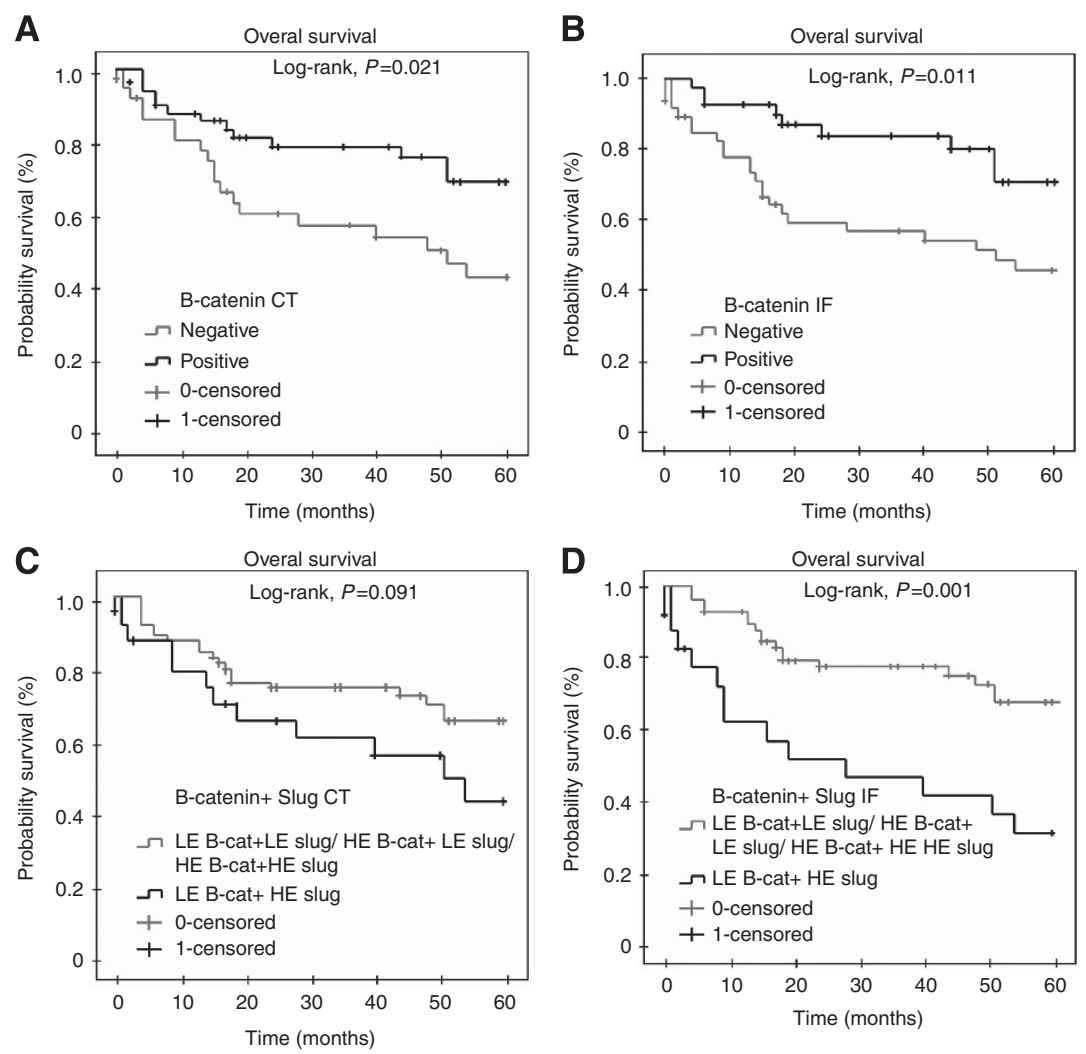

Figure 3. Overall survival analysis of 87 cases of VSCC. Shorter overall survival was observed for patients whose tumours lacked $\beta$-catenin expression at the central tumour $(P=0.021 ; \mathrm{A})$ and invasive front $(P=0.011 ; \mathrm{B})$. Overall survival curve of patients whose tumours expressed low $\beta$-catenin and high Slug at center tumour $(C)$ and invasion front $(P=0.001 ; D)$. Kaplan-Meier method, log-rank test. 

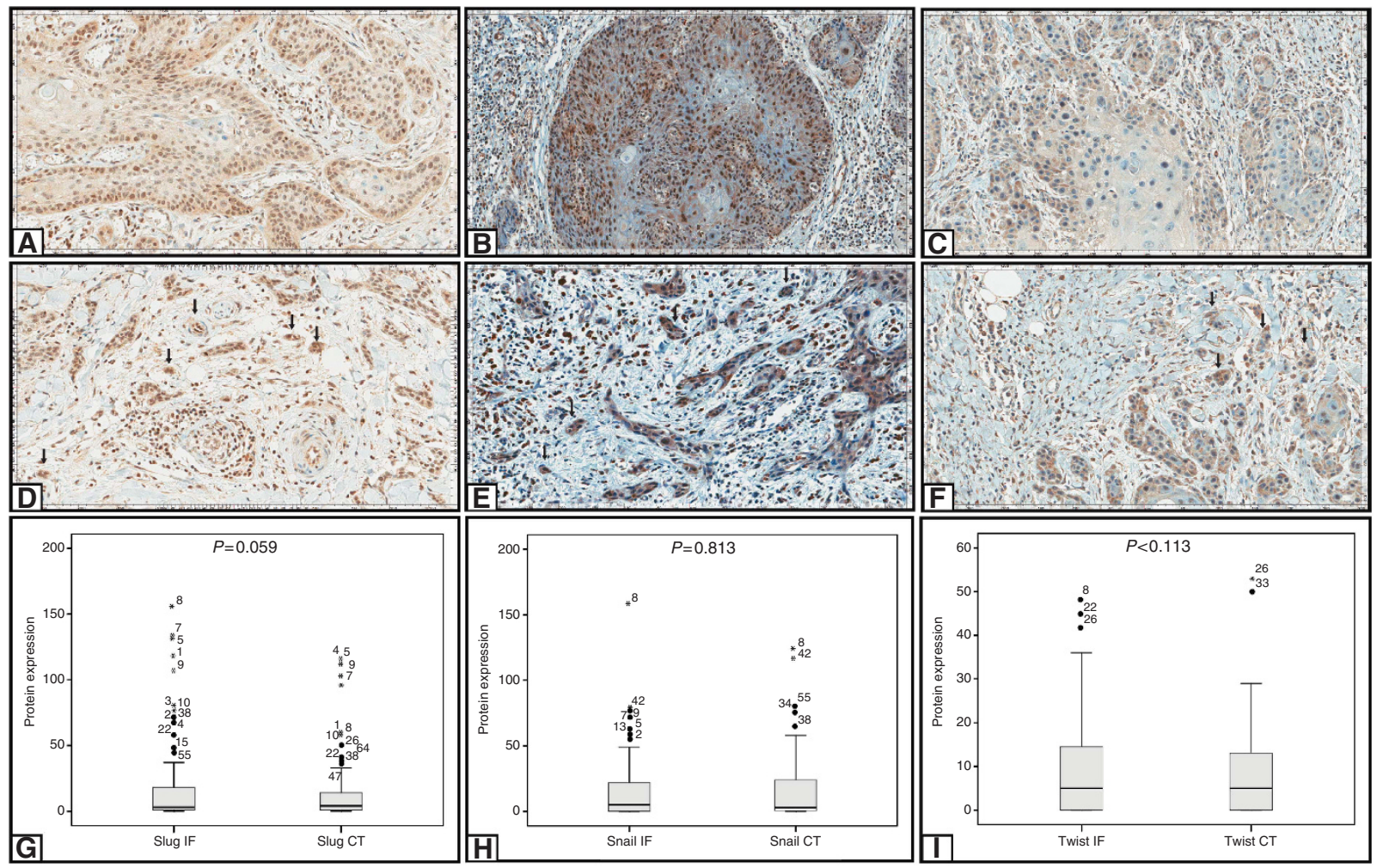

Figure 4. Immunohistochemical staining for Slug, Snail and Twist is shown at central tumour and invasive front. Positive immunostaining for Slug at central tumour (A) and invasive front (D); positive immunostaining for Snail at central tumour (B) and invasive front (E); positive immunostaining for Twist at central tumour $(\mathbf{C})$ and invasive front $(\mathbf{F})$. Comparison between the expression of Slug $(\mathbf{G})$, Snail $(\mathbf{H})$ and Twist (I) in central tumour and invasive front. Images A, B, D, E and F were captured at $\times 200$ magnification. Image $\mathbf{C}$ was captured at $\times 400$ magnification. ${ }^{*}$ Statistical significance, $P \leqslant 0.05$.

Table 7. $\beta$-catenin and $\beta$-catenin/Slug expression vs HPV infection

\begin{tabular}{|c|c|c|c|c|}
\hline $\begin{array}{l}\text { Protein } \\
\text { expression }\end{array}$ & Region & Category & $\begin{array}{l}\text { HPV positive } \\
\text { N/total (\%) }\end{array}$ & $\boldsymbol{P}$-value \\
\hline \multirow[t]{4}{*}{$\beta$-catenin ${ }^{\text {low }}$} & IF & No & $37 / 53(69.8)$ & $0.000^{a}$ \\
\hline & & Yes & $10 / 34(29.4)$ & \\
\hline & CT & No & $30 / 53(56.6)$ & $0.001^{a}$ \\
\hline & & Yes & $7 / 34(20.6)$ & \\
\hline \multirow[t]{4}{*}{$\beta$-catenin ${ }^{\text {low }} /$ slug $^{\text {high }}$} & IF & No & $21 / 53(39.6)$ & $0.000^{a}$ \\
\hline & & Yes & $2 / 34(5.9)$ & \\
\hline & CT & No & $21 / 53(39.6)$ & $0.002^{a}$ \\
\hline & & Yes & $3 / 34(8.8)$ & \\
\hline
\end{tabular}

diffuse immunostaining pattern was observed. Furthermore, the majority of previous studies indicated that loss of E-cadherin expression has a close correlation with metastasis (Sawada et al, 2008; von Burstin et al, 2009; Tang et al, 2011). In the light of ours and other results, it is suggestive that E-cadherin expression may be lower in primary tumours that can potentially progress to lymph node or distant metastasis.

Association between E-cadherin and $\beta$-catenin is known to be essential for proper anchorage to the cytoskeleton and is necessary for E-cadherin-binding function (Thiery et al, 2009). Our results have demonstrated that the loss of $\beta$-catenin expression strongly characterises the transition from cells at the central tumour to invasive nests or isolated tumour cells in elderly patients with VSCC having no HPV infection (Table 7). Similar findings have
Table 8. Correlation between $\beta$-catenin expression and clinical data of patients with vulvar carcinoma

\begin{tabular}{|l|c|c|c|c|}
\hline Variables & $\mathbf{N}$ & $\mathbf{P}$-value & $\begin{array}{c}\text { Hazard ratio } \\
\text { for survival }\end{array}$ & $\mathbf{9 5 . 0 \%} \mathrm{Cl}$ \\
\hline $\begin{array}{l}\text { Vascular and } \\
\text { perineural invasion }\end{array}$ & 19 & 0.204 & 1.650 & $0.762-3.569$ \\
\hline FIGO stages & 30 & 0.577 & 1.264 & $0.555-2.881$ \\
\hline HPV & 53 & 0.367 & 1.493 & $0.625-3.564$ \\
\hline $\begin{array}{l}\beta \text {-catenin central } \\
\text { tumour }\end{array}$ & 37 & 0.928 & 1.047 & $0.386-2.841$ \\
\hline $\begin{array}{l}\beta \text {-catenin invasion } \\
\text { front }\end{array}$ & 47 & $0.044^{\mathrm{a}}$ & 2.131 & $1.022-5.234$ \\
\hline
\end{tabular}

Abbreviations: $\mathrm{Cl}=$ confidence interval; $\mathrm{FIGO}=$ International Federation of Gynecology and Obstetrics; HPV = human papillomavirus. multivariate analysis $(N=87)$

a Statistically significant, $P \leqslant 0.05$

been recorded in cervical squamous carcinoma, in which there was reduced $\beta$-catenin immunoexpression in many of infiltrative tumour cells, particularly at the tumour-stromal interface (Koay et al, 2012). In the present study, loss of $\beta$-catenin expression was statistically associated with FIGO stages III and IV, and Cox regression model also demonstrated that loss $\beta$-catenin expression was an independent factor associated with poor survival (Table 8). Previous studies demonstrated that $\beta$-catenin is an important marker of invasion for cervical neoplasms, and therefore can be useful in the diagnostic setting (Stewart et al, 2011). Our results allow us to confirm these findings and also to suggest that the assessment of $\beta$-catenin expression by IHC at the invasive tumour front may represent an important tool in establishing prognosis of patients with vulvar cancer. 


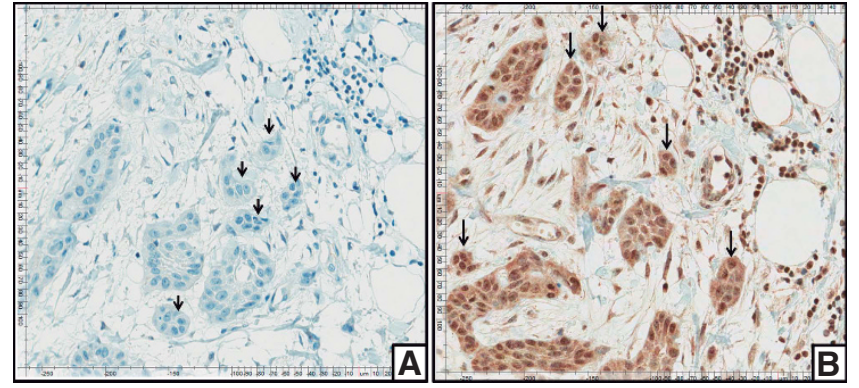

Figure 5. Immunohistochemical staining for Slug and $\boldsymbol{\beta}$-catenin. Negative immunostaining for $\beta$-catenin at invasive front (A). Positive immunostaining for Slug at invasive front (B).

In our series, loss of $\beta$-catenin expression was associated with a significant increase in the expression of Slug and Vimentin at the invasive front when compared with central tumour. Loss of expression of $\beta$-catenin and gain of Slug could be a key feature of EMT-like events, and therefore the current findings could suggest that EMT-like has an important role in invasion of VSCC. Kojc et al (2009) showed that upregulation of transcription repressors Snail, Slug and Twist on both mRNA and protein levels presumably results in transient EMT, which has been postulated to be responsible for tumour progression and metastasising (Yuen et al, 2007). Interestingly, our results show strong association between high expression of Slug, Snail and Twist2 at the invasive front with negative HPV cases (Table 5). Furthermore, the strongest association was between $\beta$-catenin ${ }^{\text {low }} /$ Slug $^{\text {high }}$ tumours with HPV-negative, late stage and patients with poorest overall survival. Other data of our group are consistent with the fact that vulvar tumours associated with HPV infection, which has been shown to be an independent predictor of better survival, have a better prognosis compared with tumours with p53 mutations (Kumar et al, 2009; De Melo Maia et al, 2013). Furthermore, our results are in agreement with these studies, as our HPV-positive cases showed better overall survival than those of HPV-negative ones; however, the multivariate analysis did not show an independent prognostic significance in these cases.

These observations led us to hypothesise that those alterations in $\beta$-catenin and Slug expression may characterise a potentially more aggressive behaviour of the tumour cells in the tumour front, with increased risk of deeper invasion and metastasis. These results, together with other results from our group, drive us to conclude that HPV-positive tumours do not show evidences of EMT-like events, with usually better prognosis (De Melo Maia et al, 2012; Lavorato-Rocha et al 2013). On the other hand, the HPV-negative tumours develop EMT-like and, therefore increased capability of invasion and progression, leading to worse prognosis and poorer outcome.

The metastatic process of epithelial tumours is characterised by alteration in E-cadherin and $\beta$-catenin expression, and seems to occur at different time points, depending on the patient's HPV status. Al Moustafa et al (2004) showed that overexpression of E6()E7 in combination with ErbB-2 activation downregulates E-cadherin and membranous $\beta$-catenin expression. This is accompanied by nuclear translocation of $\beta$-catenin with subsequent upregulation of oncoproteins responsible for tumour progression (Al Moustafa et al, 2004). In our series, besides loss of $\beta$-catenin being related with negativity for HPV infection (Table 7), no nuclear $\beta$-catenin was detected at all, suggesting that other pathways apart from HPV-related Wnt activation may be related with EMT-like events in vulvar cancer.

Vimentin is another upregulated protein in aggressive tumour phenotypes related to EMT phenomenon (Brabletz et al, 2005; Otsuki et al, 2011). Vimentin is ubiquitously expressed by cells of mesenchymal origin including fibroblasts, endothelial cells, smooth muscle cells and leucocytes (Mor-Vaknin et al, 2003). Our results indicated that the invasive front expressed higher Vimentin than that of central tumour. Furthermore, Vimentin immunopositivity was also significantly associated with invasion depth and associated lesions (lichen sclerosus and squamous hyperplasia; Table 3A). De Araujo et al (1993) demonstrated that the expression of the Vimentin was most marked in oral squamous cell carcinoma with high tumour grade. Also, significant correlation between Vimentin expression and aggressive tumour features, including lymph node involvement, was recently shown by immunophenotypic analysis of the head and neck squamous cell carcinoma (Mandal et al, 2008; Dal Vechio et al, 2011). Our results did not indicate significant association between Vimentin expression and HPV infection.

In conclusion, E-cadherin, $\beta$-catenin, Slug, Snail, Twist 2 and Vimentin expression are important features for the characterisation of EMT-like events, known to be related to invasive and metastatic phenotype in many tumours, including vulvar cancer. According to our results, loss of E-cadherin expression is observed in advanced stages of tumour invasion. $\beta$-catenin represents an important biomarker for establishing prognosis in vulvar cancer, as its loss of expression is related to poorer outcome, even in the multivariate analysis. Furthermore, $\beta$-catenin lower expression associated with gain in Slug expression characterises a subgroup of EMT-related HPV-negative tumours with the worst outcome, increased invasiveness and progression. Human papillomaviruspositive tumours did not exhibit EMT-like events, and had better prognosis. In the clinical setting, IHC-comparative assessment of $\beta$-catenin expression between invasive front and central tumour may represent an additional tool for establishing prognosis of vulvar cancer.

\section{ACKNOWLEDGEMENTS}

We gratefully thank Fundação de Amparo à Pesquisa do Estado de São Paulo (FAPESP) for financial support.

\section{CONFLICT OF INTEREST}

The authors declare no conflict of interest.

\section{REFERENCES}

Alvarenga AW, Coutinho-Camillo CM, Rodrigues BR, Rocha RM, Torres LF, Martins VR, da Cunha IW, Hajj GN (2013) A comparison between manual and automated evaluations of tissue microarray patterns of protein expression. J Histochem Cytochem 61: 272-282.

Al Moustafa AE, Foulkes WD, Benlimame N, Wong A, Yen L, Bergeron J, Batist G, Alpert L, Alaoui-Jamali MA (2004) E6/E7 proteins of HPV type 16 and ErbB-2 cooperate to induce neoplastic transformation of primary normal oral epithelial cells. Oncogene 23: 350-358.

Bhangu A, Wood G, Mirnezami A, Darzi A, Tekkis P, Goldin R (2012) Epithelial mesenchymal transition in colorectal cancer: seminal role in promoting disease progression and resistance to neoadjuvant therapy. Surg Oncol 21: 316-323.

Brabletz T, Hlubek F, Spaderna S, Schmalhofer O, Hiendlmeyer E, Jung A, Kirchner T (2005) Invasion and metastasis in colorectal cancer: epithelialmesenchymal transition, mesenchymal-epithelial transition, stem cells and beta-catenin. Cells Tissues Organs 179: 56-65.

Cardiff RD (2005) Epithelial to mesenchymal transition tumors: fallacious or snail's pace? Clin Cancer Res 11: 8534-8537.

Carico E, Radici M, Losito NS, Raffa S, Firrisi L, Fabiano A, Manola M, Vecchione A, Giovagnoli MR (2012) Expression of E-cadherin and $\alpha$-catenin in T1 N0 laryngeal cancer. Anticancer Res 32: 5245-5249.

Cooke VG, LeBleu VS, Keskin D, Khan Z, O'Connell JT, Teng Y, Duncan MB, Xie L, Maeda G, Vong S, Sugimoto H, Rocha RM, Damascena A, Brentani 
RR, Kalluri R (2012) Pericyte depletion results in hypoxia-associated epithelial-to-mesenchymal transition and metastasis mediated by met signaling pathway. Cancer Cell 21: 66-81.

Dal Vechio AM, Giudice FS, Sperandio FF, Mantesso A, Pinto Junior Ddos S (2011) Vimentin expression and the influence of Matrigel in cell lines of head and neck squamous cell carcinoma. Braz Oral Res 25: 235-240.

De Araujo VC, Pinto Júnior DS, de Sousa SO, Nunes FD, de Araujo NS (1993) Vimentin in oral squamous cell carcinoma. Eur Arch Otorhinolaryngol 250: 105-109.

De Melo Maia B, Munhoz Cestari F, Lavorato-Rocha AM, Sant'ana Rodrigues I, Baiocchi G, Cardoso Guimarães G, Vassallo J, Stiepcich M, Soares FA, Malagoli Rocha R (2013) Characterization of sociodemographic and clinicopathological features in brazilian patients with vulvar squamous cell carcinoma. Gynecol Obstet Invest 75: 53-60.

De Melo Maia B, Lavorato-Rocha AM, Rodrigues IS, Baiocchi G, Cestari FM, Stiepcich MM, Chinen LT, Carvalho KC, Soares FA, Rocha RM (2012) Prognostic significance of c-KIT in vulvar cancer: bringing this molecular marker from bench to beside. J Transl Med 10: 150.

Heuberger J, Birchmeier W (2010) Interplay of cadherin-mediated cell adhesion and canonical Wnt signaling. Cold Spring Harb Perspect Biol 2: a002915.

Koay MHE, Crook M, Stewart CJR (2012) Cyclin D1, E-cadherin and betacatenin expression in FIGO stage IA: diagnostic value and evidence for epithelial-mesenchymal transition. Histopathology 61: 1125-1133.

Kojc N, Zidar N, Gale N, Poljak M, Fujs Komlos K, Cardesa A, Höfler H, Becker KF (2009) Transcription factors Snail, Slug, Twist, and SIP1 in spindle cell carcinoma of the head and neck. Virchows Arch 454: 549-555.

Kumar S, Shah JP, Bryant CS, Imudia AN, Morris RT, Malone JM (2009) A comparison of younger vs older women with vulvar cancer in the United States. Am J Obstet Gynecol 200: 52-55.

Lanneau GS, Argenta PA, Lanneau MS, Riffenburgh RH, Gold MA, McMeekin DS, Webster N, Judson PL (2009) Vulvar cancer in young women: demographic features and outcome evaluation. Am J Obstet Gynecol 200(645): e1-e5.

Lavorato-Rocha AM, De Melo Maia B, Rodrigues IS, Stiepcich MM, Baiocchi G, Cestari FM, Carvalho KC, Soares FA, Rocha RM (2013) Prognostication of vulvar cancer based on p14(ARF) status: molecular assessment of transcript and protein. Ann Surg Oncol 20: 31-39.

Mandal M, Myers JN, Lippman SM, Johnson FM, Williams MD, Rayala S, Ohshiro K, Rosenthal DI, Weber RS, Gallick GE, El-Naggar AK (2008) Epithelial to mesenchymal transition in head and neck squamous carcinoma: association of Src activation with E-cadherin down-regulation, vimentin expression, and aggressive tumor features. Cancer 112: 2088-2100.

Mor-Vaknin N, Punturieri A, Sitwala K, Markovitz DM (2003) Vimentin is secreted by activated macrophages. Nat Cell Biol 5: 59-63.

Otsuki S, Inokuchi M, Enjoji M, Ishikawa T, Takagi Y, Kato K, Yamada H, Kojima K, Sugihara K (2011) Vimentin expression is associated with decreased survival in gastric cancer. Oncol Rep 25: 1235-1242.
Paterson EL, Kazenwadel J, Bert AG, Khew-Goodall Y, Ruszkiewicz A, Goodall GJ (2013) Down-regulation of the miRNA-200 family at the invasive front of colorectal cancers with degraded basement membrane indicates EMT is involved in cancer progression. Neoplasia 15 : 180-191.

Peinado H, Olmeda D, Cano A (2007) Snail, Zeb and bHLH factors in tumour progression: an alliance against the epithelial phenotype? Nat Rev Cancer 7: 415-428.

Santeufemia DA, Capobianco G, Re GL, Miolo GM, Fadda GM, Cherchi PL, Tumolo S (2012) Cisplatin-gemcitabine as palliative chemotherapy in advanced squamous vulvar carcinoma: report of two cases. Eur J Gynaecol Oncol 33: 421-422.

Sawada K, Mitra AK, Radjabi AR, Bhaskar V, Kistner EO, Tretiakova M, Jagadeeswaran S, Montag A, Becker A, Kenny HA, Peter ME, Ramakrishnan V, Yamada SD, Lengyel E (2008) Loss of E-cadherin promotes ovarian cancer metastasis via alpha 5-integrin, which is a therapeutic target. Cancer Res 68: 2329-2339.

Stewart CJ, Crook ML, Little L, Louwen K (2011) Correlation between invasive pattern and immunophenotypic alterations in endocervical adenocarcinoma. Histopathology 58: 720-728.

Sun S, Ning X, Zhang Y, Lu Y, Nie Y, Han S, Liu L, Du R, Xia L, He L, Fan D (2009) Hypoxia-inducible factor-1alpha induces Twist expression in tubular epithelial cells subjected to hypoxia, leading to epithelial-tomesenchymal transition. Kidney Int 75: 1278-1287.

Tang B, Peng ZH, Yu PW, Yu G, Qian F (2011) Expression and significance of $\mathrm{Cx} 43$ and E-cadherin in gastric cancer and metastatic lymph nodes. Med Oncol 28: 502-508.

Thiery JP, Acloque H, Huang RY, Nieto MA (2009) Epithelial-mesenchymal transitions in development and disease. Cell 139: 871-890.

van de Nieuwenhof HP, Bulten J, Hollema H, Dommerholt RG, Massuger LF, van der Zee AG, de Hullu JA, van Kempen LC (2011) Differentiated vulvar intraepithelial neoplasia is often found in lesions, previously diagnosed as lichen sclerosus, which have progressed to vulvar squamous cell carcinoma. Mod Pathol 24: 297-305.

von Burstin J, Eser S, Paul MC, Seidler B, Brandl M, Messer M, von Werder A, Schmidt A, Mages J, Pagel P, Schnieke A, Schmid RM, Schneider G, Saur D (2009) E-cadherin regulatesmetastasis of pancreatic cancer in vivo and is suppressed by a SNAIL/HDAC1/HDAC2 repressor complex. Gastroenterology 137: 361-371.

Yuen HF, Chua CW, Chan YP (2007) Significance of TWIST and E-cadherin expression in the metastatic progression of prostatic cancer. Histopathology 50: 648-658.

This work is published under the standard license to publish agreement. After 12 months the work will become freely available and the license terms will switch to a Creative Commons AttributionNonCommercial-Share Alike 3.0 Unported License.

Supplementary Information accompanies this paper on British Journal of Cancer website (http://www.nature.com/bjc) 